\title{
Granular computing in decision-making
}

\author{
Jindong Qin ${ }^{1} \cdot$ Xinwang Liu² ${ }^{2}$ Luis Martínez ${ }^{3}$
}

Published online: 23 May 2018

๑) Springer International Publishing AG, part of Springer Nature 2018

\section{Introduction}

Granular computing presents a coherent framework using concepts like information granules and levels or hierarchies of information for dealing with knowledge acquired and processed at different levels of abstraction. The concept of information granularity occurs in a wide variety of fields. During the last decade, granular computing has emerged as a new asset of study human-centric decision-making problem. Because it supports a general sequence of processing that can be depicted as: data/experimental evidence, information granules, and constructs of decision-making. This is due to the fact that granular computing is often applied to classical uncertainty-related areas, such as interval analysis, fuzzy sets, rough sets, and shadow sets. The studies in decisionmaking with the aid of granular computing are promising and should be further researched to develop new theories, methodologies, and tools.

This Special Issue is devoted to granular computing in decision-making that represents an important advance in the topic from the theoretical and practical points of view, to apply granular computing in decision-making processes for acquiring, representing, processing information in such processes.

This Special Issue encompasses five high-quality papers focused on different related topics falling within the scope of this project.

The first paper, An integrated ANP-VIKOR methodology for sustainable supplier selection with interval type-2 fuzzy sets by Liu et al., introduces and integrated analytical

Jindong Qin

qinjindongseu@126.com

1 School of Management, Wuhan University of Technology, Wuhan 430070, Hubei, China

2 School of Economics and Management, Southeast University, Nanjing 210096, Jiangsu, China

3 Department of Computer Science, University of Jaén, Jaén 23071, Spain network process (ANP) method with the ViseKriterijumska Optimizacija I Kompromisno Resenje (VIKOR) method under interval type-2 fuzzy environment to solve supplier selection in sustainable supply chain management.

Mei and Xie introduce the determination of an optimal evacuation strategy for improving the evacuation process efficiency when an emergency event happens in a Metro station in the paper Evacuation Strategy of Emergent Event in Metro Station based on the ELECTRE Method. They propose a ELECTRE group decision-making process which models the elicited information by experts using triangular intuitionistic fuzzy numbers. Such a model is put into practice by assuming a fire emergency event in a metro station in the city of Wuhan, China.

Subsequently, Liu and Liang present the paper Multigranularity unbalanced linguistic group decision making with incomplete weight information based on VIKOR method, in which a novel group decision-making model based on VIKOR method is introduced. It models the preferences elicited by experts with different background and different levels of knowledge about the problem by means of multi-granular unbalanced linguistic information. Such a decision model is applied to a green supplier selection.

Zulueta and Sanchez presents the paper Linguistic Dynamic Multicriteria Decision Making using Symbolic Linguistic Computing Models in which different linguistic symbolic aggregation operators are extended throughout time-dependent operators able to operate with multi-period linguistic information and obtain accurate and understandable results in linguistic dynamic multi-criteria decisionmaking problems. Finally, a green supplier selection problem is developed.

The last paper, A Choquet integral based approach to multiattribute decision making with correlated periods is a theoretical study in which Zulueta-Veliz and GarciaCabrera use fuzzy linguistic granules to model information in dynamic multi-attribute decision making with the aim of managing decision making processes that are subject to correlated periods. Therefore, in order to aggregate the different fuzzy linguistic information gathered in multiple periods 
whose importance are interdependent, different dynamic aggregation operators (correlated averaging and correlated geometric) are developed based on the Choquet integral. Eventually, a multi-attribute decision model that includes these operators is presented and an illustrative example demonstrates the effectiveness of these operators in a practical environment.

We really hope that this Special Issue will demonstrate and stimulate the field to address new research challenges for granular computing in decision-making.

The Guest Editors thank all the contributors for their effort in submitting high-quality papers, all the reviewers for their careful and constructive comments to further enhance the quality of the papers, and Prof. W. Pedrycz and Prof. S.-M. Chen, Editors-in-chief of Granular Computing, for their valuable advice, support and assistance in the organization of this Special Issue.

Jindong Qin

Xinwang Liu

Luis Martínez

Publisher's Note Springer Nature remains neutral with regard to jurisdictional claims in published maps and institutional affiliations. 